\title{
D-brane stability, geometric engineering, and monodromy in the Derived Category
}

\author{
Robert L. Karp* \\ Department of Physics, Rutgers University \\ 136 Frelinghuysen Rd., Piscataway, NJ 08854 USA
}

\begin{abstract}
We discuss aspects of topological B-type D-branes in the framework of the derived category of coherent sheaves $\mathbf{D}(X)$ on a Calabi-Yau 3-fold $X$. We analyze the link between massless D-branes and monodromies in the CFT moduli space. A classification of all massless D-branes at any point in the moduli space is conjectured, together with an associated monodromy. We test the conjectures in two independent ways. First we establish a composition formula for certain Fourier-Mukai functors, which is a consequence of the triangulated structure of $\mathbf{D}(X)$. Secondly, using $\pi$-stability we rederive the stable soliton spectrum of the pure $\mathcal{N}=2$ supersymmetric $\mathrm{SU}(2)$ SeibergWitten theory. In this approach, the simplicity of the spectrum rests on Grothendieck's theorem concerning vector bundles over $\mathbb{P}^{1}$.
\end{abstract}

${ }^{*}$ Email: karp@physics.rutgers.edu 


\section{Background}

The original idea that a D-brane is a subspace in target space where open strings can end is too simplistic. In the case of closed strings we know that even at $g_{s}=0$ there are $\alpha^{\prime}-$ corrections which modify the classical geometry. Accordingly, one expects that the picture of D-branes as vector bundles over submanifolds is to be altered as well.

On a Calabi-Yau n-fold $X$, there are two types of boundary conditions $[1,2]$, and hence D-branes: A-type and B-type. To gain additional control one can consider a suitable topologically twisted version of the boundary non-linear sigma model. The topological twisting can itself be of A-type or B-type. Moreover, A-type branes are compatible with the A-twist, while B-type branes are compatible with the B-twist. For topological D-branes the following picture emerges:

- A-type - wrapping Lagrangian real n-cycles, and giving rise to objects in the derived Fukaya category of Lagrangian submanifolds;

- B-type - wrapping holomorphic cycles, and giving rise to objects in the bounded derived category of coherent sheaves $\mathbf{D}(X)$ of $X$.

The homological mirror symmetry conjecture of Kontsevich gives a categorical equivalence between these two triangulated $A_{\infty}$-categories. The conjecture has already been proved for elliptic curves, and recently for quartic K3-surfaces [3].

In this paper we focus on B-branes, where using mirror symmetry, all the $\alpha^{\prime}$-corrections can be understood exactly [4]. But now, instead of physical B-branes, living in the boundary conformal field theory, we have topological B-branes, and a priori it is not clear what is relationship between them. This is where the notion of П-stability pioneered by Douglas et al. $[5,6]$ comes into play: a topological B-brane is physical if it is $\Pi$-stable. We will have more to say about $\Pi$-stability, but first we have to review some terminology. For the precise definition of $\Pi$-stability the reader should consult for example [4].

Given two D-branes, $A$ and $B$, the totality of topological open strings stretching between them is represented by $\operatorname{Hom}(\mathrm{A}[n], \mathrm{B})$, for any integer $n$. Note that for a D-brane $\mathrm{A}, \mathrm{A}[1]$ represents its anti-brane.

The most interesting open strings are in $\operatorname{Hom}(A[-1], B)$. Giving vev to such a string forms a potential bound state, depending on whether the open string is tachyonic or not. Mathematically this gives us a cone, $\operatorname{Cone}(f: \mathrm{A}[-1] \rightarrow \mathrm{B})$, and a distinguished triangle:

$$
\mathrm{A}[-1] \rightarrow \mathrm{B} \rightarrow \text { Cone }(f) \rightarrow \mathrm{A} .
$$

This construction brings democracy to bound states: any vertex of this triangle is a potential bound state of the other two vertices.

Each stable D-brane is given a grade $\varphi \in \mathbb{R}$ which varies continuously over the moduli space, although not in a single-valued way $[5,6]: \varphi(\mathrm{A})=-\arg (Z(\mathrm{~A})) / \pi$. Near the large radius limit the central charge is

$$
Z(\mathrm{~F})=\int_{X} \mathrm{e}^{B+i J} \operatorname{ch}(\mathrm{F}) \sqrt{t d(X)}+\ldots \text { (quantum corrections). }
$$


Using mirror symmetry we can in fact evaluate all the quantum corrections [4]. Then the mass of an open string becomes exactly computable as well: $M_{\mathrm{A}[-1] \rightarrow \mathrm{B}}^{2} \sim \varphi(\mathrm{B})-\varphi(\mathrm{A})$. It is $M_{\mathrm{A}[-1] \rightarrow \mathrm{B}}^{2}$ that determines the stability of Cone $(f)$ in (1), whether it is a bound state or not.

\section{Monodromy}

To learn more about the behavior of D-branes in non-trivial backgrounds, we will subject them to monodromy transformations. Henceforth we follow the set of stable D-branes as we traverse a loop in the moduli space of complexified Kahler forms. The interesting loops are those that cross a line of marginal stability for some D-brane B, causing it to decay, and those that encircle points in moduli space where some D-brane A becomes massless. To restore the physics we had before looping we are required to relabel the D-branes. This relabeling is the monodromy action on D-branes. Note that monodromy is not a statement about a certain D-brane manifestly becoming another D-brane, but rather it is the relabeling process.

A good place to start looping is the discriminant locus $\Delta$. This is the subvariety in the moduli space of complexified Kahler forms where the associated conformal field theory is singular. Alternatively, it is the locus in the moduli space of complex structures of the mirror Calabi-Yau where this latter one becomes singular.

The paradigmatic example of monodromy is given by the following

Conjecture 1 (Kontsevich, Horja, Morrison) The monodromy action for looping around a component of the discriminant locus where a single D-brane A becomes massless is: $\mathrm{B} \longmapsto$ $\operatorname{Cone}(\operatorname{hom}(A, B) \otimes A \rightarrow B)$.

This monodromy can be understood physically, at least for some branes B, as B splitting off A[1]'s a certain number of times [7]. Naturally the question arises: what could A be? An instance is given by

Conjecture 2 At a generic point on the primary component of the discriminant locus $\Delta$, it is the D6-brane $\mathcal{O}_{X}$ wrapping the 3-fold, and its translates, that become massless. At a generic point no other D-branes become massless.

But can we do better, deal with more interesting degenerations? Maybe several D-branes becoming massless? Possibly infinitely many becoming massless? The typical situation as we approach a wall of the Kähler cone is that some subspace $E$ of the Calabi-Yau $X$ shrinks to a subspace $Z$ :

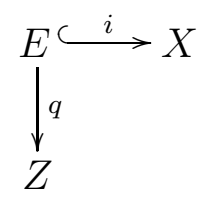

Following [8] we call this an EZ-degeneration. In this case the following conjecture is known: 
Conjecture 3 [7] Any D-Brane which becomes massless at a point on the discriminant associated with an EZ-degeneration is generated by objects of the form $i_{*} q^{*} \mathbf{z}$ for $\mathbf{z} \in \mathbf{D}(Z)$.

In such a case, there is usually a rational curve $\mathbb{P}^{1}$ in the moduli space, connecting the two limit points: the large radius limit point with the EZ-point. The EZ-point corresponds to the phase reached by following the EZ-degeneration through the wall of the Kahler cone. Regarding this situation we have the following

Conjecture $4 \quad[7,8]$ The monodromy around the discriminant inside the above $\mathbb{P}^{1}$ is the following autoequivalence of the derived category of coherent sheaves on $X$, i.e. of $\mathbf{D}(X)$ : $\mathrm{B} \mapsto \operatorname{Cone}\left(i_{*} q^{*} q_{*} i^{!} \mathrm{B} \rightarrow \mathrm{B}\right)$.

In what follows, we present two different ways of checking the consistency of these conjectures. The first example is based upon the fact that a certain monodromy can be computed in two different ways. Firstly by making use of the Conjectures, and secondly by exploring the simple structure of loops on marked $\mathbb{P}^{1}$ 's. The second example, based on geometric engineering, will also probe aspects of $\Pi$-stability. Nothing we say here is new, most of what follows can be found in [7] and [9]. The reader is also referred to these papers for most of the details.

\section{Consistency checks}

\subsection{The Model}

As a starting point we consider $X$ to be the well known degree 8 hypersurface in the resolution of $\mathbb{P}_{\{2,2,2,1,1\}}^{4}$ (see e.g. $[10,11]$ ). $X$ is then a K3-fibration $\pi: X \rightarrow C$ over a base $C \cong \mathbb{P}^{1}$, with a section. Call the generic K3 fiber $F$.

What we want is the quantum-corrected moduli space of complexified Kähler forms on $X$. This can be understood via mirror symmetry as the complex structure moduli space of the mirror $Y$. Choosing algebraic coordinates on the moduli space, the "primary" component of the discriminant is given by $\Delta_{0}=\left(1-2^{8} x\right)^{2}-2^{18} x^{2} y$. The Kähler cone is two dimensional, where $x$ controls the size of the K3-fiber, and $y$ the size of the base $C \cong \mathbb{P}^{1}$.

\subsection{Consistency of different monodromies}

We can make the base $\mathbb{P}^{1}$ very large by sending $y \rightarrow 0$. But the primary component $\Delta_{0}$ has second order intersection with $y=0$, call this point $P$. The $y=0$ curve is rational, and has three distinguished points: the large Calabi-Yau limit point $(x=y=0)$, the hybrid "P1-phase" point, and $P$.

We want to analyze the monodromy around $P$. The transition associated to $P$ is of EZ-type, and consists of collapsing $X$ onto the $\mathbb{P}^{1}$ base: $E=X, Z=\mathbb{P}^{1}, i=i d_{X}$ and $q=\pi$. Using Conjecture 4 we can immediately deduce the monodromy action: $\mathrm{B} \mapsto \boldsymbol{H}(\mathrm{B})=$ Cone $\left(\pi^{*} \pi_{*} \mathrm{~B} \rightarrow \mathrm{B}\right)$. 
On the other hand singularity theory allows us to write the monodromy around $P$ in a completely different way (see e.g. [12]), as $\boldsymbol{L}^{-1} \boldsymbol{K} \boldsymbol{L} \boldsymbol{K}$, where $\boldsymbol{L}$ is the large radius monodromy $\boldsymbol{L}(\mathrm{B})=\mathrm{B} \otimes \mathcal{O}_{X}(F)$, while $\boldsymbol{K}$ is the Kontsevich et al. monodromy of Conjecture 1: $\boldsymbol{K}(\mathrm{B})=$ Cone $\left(\operatorname{hom}\left(\mathcal{O}_{X}, \mathrm{~B}\right) \otimes \mathcal{O}_{X} \rightarrow \mathrm{B}\right)$.

By making extensive use of the structures in the derived categories of $X$ and $\mathbb{P}^{1}$, it was shown in [7] that $\boldsymbol{H}=\boldsymbol{L}^{-1} \boldsymbol{K} \boldsymbol{L} \boldsymbol{K}$ indeed holds, providing a strong consistency check for the monodromy conjectures.

\subsection{Geometric engineering}

Now we turn to the second test of our conjectures. We can use the techniques of $[13,14]$ to engineer an $N=2$ pure $\mathrm{SU}(2)$ gauge theory using the above mentioned Calabi-Yau $X$ [9]. The goal is to understand the stable BPS solitons spectrum of the Yang-Mills theory $[15,16]$ from the П-stable BPS D-branes spectrum of the Type IIA theory.

The geometric engineering limit requires again the base $C$ to be very large, $y \rightarrow 0$, and we must also lie very close to the discriminant locus. Note that the second order intersection point $\left(2^{-8}, 0\right)$ splits for $y=\epsilon$. Introducing the variable $u$ by

$$
u^{-2}=4 y /\left(2^{8} x-1\right)^{2}
$$

places the two intersection points at $u= \pm 1$. In this limit the Picard-Fuchs equations satisfied by the periods on $X$ reduce to the one satisfied by the Seiberg-Witten periods [9]:

$$
\left(1-u^{2}\right) \partial_{u}^{2} a-2 u \partial_{u} a-\frac{1}{4} a=0 .
$$

But it is precisely the periods that together with the D-brane charges determine the central charges, and hence the stability of solitons.

The D-brane charge is measured by K-theory: $K^{*}\left(\mathbb{P}^{1}\right)=H^{0}(C) \oplus H^{2}(C)=\mathbb{Z}^{2}$. For convenience take $\mathcal{O}_{C}$ and $\mathcal{O}_{p}$, which also generate $\mathbf{D}(C)$, as the generators of $K^{*}\left(\mathbb{P}^{1}\right)$. Therefore, we require only two basic central charges: $a=Z\left(\pi^{*} \mathcal{O}_{p}\right), a_{D}=Z\left(\pi^{*} \mathcal{O}_{C}\right)$.

Conjecture 3 and and the same knot theoretic computation as in the previous subsection gives the following assignments:

- magnetic monopole: $\mathcal{O}_{X}=\pi^{*} \mathcal{O}_{C}$, i.e., the 6-brane wrapping $X$;

- dyon: $\mathcal{O}_{X}(F)=\pi^{*} \mathcal{O}_{C}(1)$, i.e., a 6-brane with 4-brane charge;

- W-boson: $\pi^{*} \mathcal{O}_{p}$, i.e., a 4-brane wrapped around a K3 fiber.

The charge assignment is in agreement with the short exact sequence of sheaves

$$
0 \rightarrow \mathcal{O}_{C}(-1) \rightarrow \mathcal{O}_{C} \rightarrow \mathcal{O}_{p} \rightarrow 0,
$$

and it's pullback to $X$. This is in itself a non-trivial fact. We can also read off the following identifications: magnetic charge $=$ rank, electric charge $=$ degree . 


\subsubsection{Weak coupling region}

For very large $|u|$ we are at the large radius limit of $X$, where all the $\alpha^{\prime}$-corrections are very small. In this limit B-type D-branes correspond to stable holomorphic vector bundles over subvarieties of $X$. Conjecture [3 allows us to work on $C \cong \mathbb{P}^{1}[9]$. For brevity we consider only the stability of rank 2 bundles.

By Grothendieck's theorem, a rank $r$ vector bundle $V$ over $C \cong \mathbb{P}^{1}$ is a direct sum of line bundles:

$$
V \cong \mathcal{O}_{C}\left(s_{1}\right) \oplus \mathcal{O}_{C}\left(s_{2}\right) \oplus \ldots \oplus \mathcal{O}_{C}\left(s_{r}\right) .
$$

Thus the general rank 2 bundles is of the form $\mathcal{O}_{C}(s) \oplus \mathcal{O}_{C}(t)$. But $\mathcal{O}_{C}(s) \oplus \mathcal{O}_{C}(t)$ fits into the triangle

$$
\mathcal{O}_{C}(s) \rightarrow \mathcal{O}_{C}(s) \oplus \mathcal{O}_{C}(t) \rightarrow \mathcal{O}_{C}(t) \rightarrow \mathcal{O}_{C}(s)[1],
$$

where $f \in \operatorname{Hom}\left(\mathcal{O}_{C}(s), \mathcal{O}_{C}(t)[1]\right)=\operatorname{Ext}^{1}\left(\mathcal{O}_{C}(s), \mathcal{O}_{C}(t)\right)$ determines the extension class.

There are two cases to consider, depending on whether $f=0$ or not, resulting in the following [9]: the rank two bundle $\mathcal{O}(s) \oplus \mathcal{O}(t)$ will decay into $\mathcal{O}\left(s^{\prime}\right) \oplus \mathcal{O}\left(t^{\prime}\right)$ for some $s^{\prime}, t^{\prime}$ such that $s^{\prime}+t^{\prime}=s+t$ and $\left|s^{\prime}-t^{\prime}\right| \leq 1$. Note that for $\mathcal{O}(s) \oplus \mathcal{O}(t)$ with $|s-t| \leq 1$, $\operatorname{Ext}^{1}\left(\mathcal{O}_{C}(s), \mathcal{O}_{C}(t)\right)=\operatorname{Ext}^{1}\left(\mathcal{O}_{C}(t), \mathcal{O}_{C}(s)\right)=0$, so there are no open strings to either stabilize or destabilize, and the two constituents are free to drift apart.

Similarly, one proves that any dyon $(r, m)$ for $r>1$ is unstable to decay into dyons with $r=1$, and there are no stable states of charge $(0, m)$ for $m>1$ either [9], in line with $[15,16]$.

\subsubsection{Strong coupling region}

By considering the distinguished triangle $\mathcal{O}_{C}(m) \rightarrow \mathcal{O}_{C}(m+1) \rightarrow \mathcal{O}_{p} \rightarrow \mathcal{O}_{C}(m)$ [1] we can show that the only stable solitons are: $\mathcal{O}_{C}$ and $\mathcal{O}_{C}(1)$, i.e., the monopole and the dyon, again in line with $[15,16]$. In particular, the $W$-boson $\mathcal{O}_{p}$ decays into $\mathcal{O}_{C}(1)$ and $\mathcal{O}_{C}[1]$ [9].

\subsubsection{Returning to weak coupling}

Suppose now that we continue our journey, back to the weakly coupled region. We would expect to recover the original set of stable solitons. But they may not be the same elements of the derived category, as they might undergo monodromy.

One would expect that as we cross the MS-line, $\mathcal{O}_{p}$ would become stable again. Unfortunately $M_{\mathcal{O}_{C} \rightarrow \mathcal{O}_{C}(1)}^{2} \sim \varphi\left(\pi^{*} \mathcal{O}_{C}(1)\right)-\varphi\left(\pi^{*} \mathcal{O}_{C}[-1]\right)$ gets larger and larger, so $\mathcal{O}_{p}$ never stabilizes. Fortunately, to rescue us there come some new bound states! Define $\mathrm{X}$ by the triangle

$$
\pi^{*} \mathcal{O}_{C}(1)[-1] \rightarrow \pi^{*} \mathcal{O}_{C}[2] \rightarrow \mathrm{X} \rightarrow \pi^{*} \mathcal{O}_{C}(1),
$$

then iterate it:

$$
\mathrm{Y}_{m} \rightarrow \mathrm{Y}_{m+1} \rightarrow \mathrm{X} \rightarrow \mathrm{Y}_{m}[1] .
$$

It turns out that all of the $\mathrm{Y}_{m}$ 's become stable as we pass the MS-line back into the weakcoupling regime. 
This infinite tower of new states $Y_{m}$ is the "replacement" for the old states $\mathcal{O}_{C}(m)$, of charge $(1, m)$. It is also true that under monodromy around $u=1$ (we can use Conjecture 1 for this) the set of states $\mathcal{O}_{C}(m)$ turn into the set of states $Y_{m}$.

But there is still an aesthetic discrepancy: the original set of weak coupling states have very clear geometric interpretation as wrapped branes, while after monodromy we have rather exotic objects. However, from the derived category point of view they look quite symmetric (see the sketch below): in the strong-coupling region we have only two stable D-branes: A and $\mathrm{B}$. As we move across the MS-line into the weak coupling regime they can form a bound state $\mathrm{W}$ which plays the rôle of the $\mathrm{W}$-boson. Crossing upwards this is done by $\mathrm{A} \rightarrow \mathrm{B}$, while crossing downwards by $B \rightarrow A[3]$. This is essentially Serre duality at work. Observe also the $\mathbf{3}$ appearing, as if the solitons in Seiberg-Witten theory knew that they should be associated with a 3 -fold!

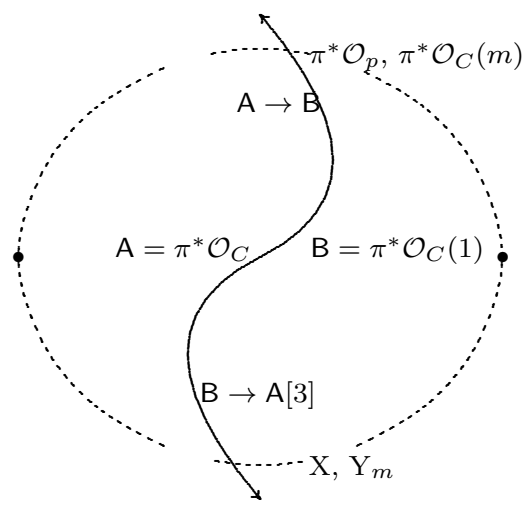

\section{Acknowledgments}

I would like to thank my advisor Paul Aspinwall for his guidance, and Paul Horja for enjoyable collaboration. This work was supported in part by the NSF grant DMS-0074072. 


\section{References}

[1] K. Becker, M. Becker, and A. Strominger, Five-branes, membranes and nonperturbative string theory, Nucl. Phys. B456 (1995) 130-152, hep-th/9507158.

[2] H. Ooguri, Y. Oz, and Z. Yin, D-branes on Calabi-Yau spaces and their mirrors, Nucl. Phys. B477 (1996) 407-430, hep-th/9606112.

[3] P. Seidel, Homological mirror symmetry for the quartic surface, math.SG/0310414.

[4] P. S. Aspinwall and M. R. Douglas, D-brane stability and monodromy, JHEP 05 (2002) 031, hep-th/0110071.

[5] M. R. Douglas, B. Fiol, and C. Romelsberger, Stability and BPS branes, hep-th/0002037.

[6] M. R. Douglas, D-branes, categories and $N=1$ supersymmetry, J. Math. Phys. 42 (2001) 2818-2843, hep-th/0011017.

[7] P. S. Aspinwall, R. L. Karp, and R. P. Horja, Massless D-branes on Calabi-Yau threefolds and monodromy, Commun. Math. Phys. 259 (2005) 45-69, hep-th/0209161.

[8] R. P. Horja, Derived category automorphisms from mirror symmetry, Duke Math. J. 127 (No. 1) (2005) 1-34, math.AG/0103231.

[9] P. S. Aspinwall and R. L. Karp, Solitons in Seiberg-Witten theory and D-branes in the derived category, JHEP 04 (2003) 049, hep-th/0211121.

[10] P. Candelas et al., Mirror symmetry for two parameter models. I, Nucl. Phys. B416 (1994) 481-538, hep-th/9308083.

[11] P. Candelas, A. Font, S. Katz, and D. R. Morrison, Mirror symmetry for two parameter models. 2, Nucl. Phys. B429 (1994) 626-674, hep-th/9403187.

[12] P. S. Aspinwall, Some navigation rules for D-brane monodromy, J. Math. Phys. 42 (2001) 5534-5552, hep-th/0102198.

[13] S. Kachru et al., Nonperturbative results on the point particle limit of $N=2$ heterotic string compactifications, Nucl. Phys. B459 (1996) 537-558, hep-th/9508155.

[14] S. Katz, A. Klemm, and C. Vafa, Geometric engineering of quantum field theories, Nucl. Phys. B497 (1997) 173-195, hep-th/9609239.

[15] N. Seiberg and E. Witten, Electric-magnetic duality, monopole condensation, and confinement in N=2 supersymmetric Yang-Mills theory, Nucl. Phys. B426 (1994) 19-52, hep-th/9407087. 
[16] F. Ferrari and A. Bilal, The Strong-Coupling Spectrum of the Seiberg-Witten Theory, Nucl. Phys. B469 (1996) 387-402, hep-th/9602082. 